${ }^{1}$ Санкт-Петербургский клинический научно-

практический

центр специализированных видов медицинской помощи (онкологический) (Санкт-Петербург, Россия)

${ }^{2}$ Санкт-Петербургский Политехнический университет Петра Великого (Санкт-Петербург, Россия)

${ }^{3}$ Научно-клинический и образовательный центр «луцевая диагностика и ядерная медицина», Санкт-Петербургский государственный университет (Санкт-Петербург, Россия)

\author{
А.А. Мелдо ${ }^{1,2}$, Л.В. Уткин ${ }^{2}$, В.М. Моисеенко ${ }^{1}$, Т.Н. Трофимова ${ }^{3}$, \\ А.А. Лукашин ${ }^{2}$, М.А. Рябинин ${ }^{2}$
}

\title{
IMPLEMENTATION OF AN INNOVATIVE APPROACH IN MEDICINE ON THE EXAMPLE OF THE INTELLECTUAL SYSTEM OF LUNG CANCER DIAGNOSIS «DOCTOR AIZIMOV»
}

\section{А.А. Мелдо ${ }^{1,2}$} Кандидатмедицинских наук, заведуюшая отделением лучевой диагностики, врач-рентгенолог СПбКНпЦСВМП(о), старший научный сотрудник, научно-исследовательская лаборатория нейросетевых технологий и искусственного интеллекта СПбПу, 197758, Санкт-Петербург, п. Песочный, Ленинградская ул., 68 А. E-mail:anna.meldo@yandex.ru.

Л.в. Уткин ${ }^{2}$ Доктор технических наук, профессор, заведующий научно-исследовательской лаборатории нейросетевых технологий и искусственного интеллекта СПбПу, 195251, Санкт-Петербург, ул. Политехническая, 29.

В.М. Моисеенко ${ }^{1}$ Доктормедицинских наук, профессор, директор СПбКНпЦСВМП(о).

T.H. Трофимова ${ }^{3}$ Доктормедицинских наук, профессор, кафедра рентгенологии и радиологии, ГБОУ ВПО ПСПбГМУ им. И.П. Павлова Минздрава России; заместитель генерального директора/ главный врач медицинской компании «АВА-Петер»; директор научно-клинического и образовательного центра «учевая диагностика и ядерная медищина» СПбГу; главный научный сотрудник Института мозга человека им. Н.П. Бехтеревой РАН;

ведущий научный сотрудник отдела әкологической физиологии Института экспериментальноймедицины; 197022, Санкт-Петербург, ул. Академика Павлова, 9.
A.A. Лукаиин ${ }^{2}$ Кандидат технических наук, начальник управления суперкомпьютерного центра, старший научный сотрудник, научно-исследовательская лаборатория нейросетевых технологий и искусственного интеллекта.

М.A. Рябинин ${ }^{2}$ Аспирант, кафедра «Телематика», инженер, научно-исследовательская лаборатория нейросетевых технологий и искусственного интеллекта.

A.A. Meldo ${ }^{1,2}$ Candidate of Medicine, Head of Radiology Department, Senior Researcher of the Laboratory of Neural Network Technologies and Artificial Intelligence,

Peter the Great Saint Petersburg Polytechnic University; Radiologist,

St. Petersburg Clinical Research Center of Specialized Types of Care (Oncology).

197758, Russia, St. Petersburg, pos. Pesochniji, Leningradskaya ul., 68A. E-mail: anna.meldo@yandex.ru.

L.V. Utkin ${ }^{2}$

Doctor of Technical Sciences, Professor, Head of the Laboratory of Neural Network Technologies and Artificial Intelligence, Peter the Great Saint Petersburg Polytechnic University, 195251, Saint Petersburg, Politecnicheskaya str., 29.

V.M. Moiseyenko ${ }^{1}$ Doctor of Medicine, Professor, Director. 


\begin{abstract}
T.N. Trofimova ${ }^{3}$
Doctor of Medicine, Professor, Radiology Department,

The First Pavlov State Medical University of St. Petersburg; Deputy of Director/Chief Physician of the medical company AVA-Peter; Director of the Scientific-Clinical and Educational Center Radiology and Nuclear Medicine, St. Petersburg State University; Senior Researcher, St. Petersburg Human Brain Institute named after N.P. Bekbtereva, Leading Researcher of the Department of Environmental Physiology, Institute of Experimental Medicine.
\end{abstract}

\author{
A.A. Lukashin ${ }^{2}$ \\ Candidate of Technical Sciences \\ Head of Supercomputer Center, \\ Senior Researcher, \\ Laboratory of Neural Network Technologies \\ and Artificial Intelligence, \\ Peter the Great Saint Petersburg Polytechnic University. \\ M.A. Ryabinin ${ }^{2}$ \\ Postgraduate Student, Engineer, \\ Laboratory of Neural Network Technologies \\ and Artificial Intelligence, \\ Peter the Great Saint Petersburg \\ Polytechnic University.
}

В статье приведено описание интеллектуальной автоматизированной системы диагностики (ИАСД) рака легкого Doctor AIzimov, которая обладает признаками различных типов инноваций (продуктовой, процессной, маркетинговой, организационной). Этот факт необходимо учитывать на этапе внедрения системы. Предлагается схема внедрения системы в практику на основе сетевой платформы, которая направлена на улучшение качества медицинской помощи, ориентирована на удовлетворение потребностей пациента и не противоречит общему устоявшемуся дизайну диагностического процесса. Данная схема является одновременно причиной и следствием постоянного усовершенствования деятельности отделения лучевой диагностики.

ключевые слова: система диагностики, рак легких, Doctor AIzimov, качество медицинской помощи, пациент, лучевая диагностика.

The article describes the computer aided diagnosis (CAD) system of lung cancer Doctor Alzimov, which has the features of different types of innovation (product, process, marketing, organizational). This fact should be taken into account in implementation of the CAD system. We present the scheme of implementation of the system in medical practice based on a network platform, which is aimed at improving the quality of medical care, focused on needs of patients and does not contradict the classic design of the diagnostic process. This scheme is a cause and a result of the continuous improvement of the radiology department.

Keywords: diagnostic system, lung cancer, Doctor Alzimov, quality of medical care, patient, radiation diagnosis.

\section{Медицина и инновации сегодня}

$\mathrm{B}$ большинстве работ, посвященных инновациям в медицине, этот процесс связывают преимущественно с поставкой нового высокотехнологичного оборудования. Внедрение инноваций в здравоохранении России - вопрос, требующий доработок. Это показано в работе [1], где авторы подчеркивают, что к наиболее значимым причинам неэффективной организации инновационной деятельности, а главное ее внедренческой составляющей отнесены: отсутствие единой, доступной базы данных по готовым инновационным продуктам, низкий уровень информационного обмена между авторами и разработчиками инноваций и практикующими врачами, консерватизм врачебного сообщества в целом, в том числе и руководителей ЛПУ, низкая инновационная культура, нежелание перемен. Тормозят процессы внедрения инноваций стандартизованная работа ЛПУ, а также отсутствие адекватного финансирования инновационной деятельности. Кроме того, авторы констатируют отсутствие действенных механизмов межведомственного, междисциплинарного взаимодействия, дисбаланс ответственности и полномочий, что мешает включению инновационной составляющей в деятельность медицинских учреждений. Таким образом, внедрение инноваций в здравоохранении России - вопрос, требующий доработок. В последние годы большое внимание уделяется созданию систем искусственного интеллекта (ИИ) в медицине, но, несмотря на многочисленные публикации, внедрение этих систем оставляет желать лучшего, поскольку проекты ограничиваются этапом научной концепции и тестированием. Вместе с тем, внедрение их в практику является важнейшей составляющей инновационной деятельности, поскольку только при этом условии инновация обеспечит формирование условий для повышения уровня производительности труда и эффективности деятельности. Кроме того, развитие искусственного интеллекта (ИИ) в целом, и в медицине в частности, может быть рассмотрено как мера реализации и обеспечения национальных интересов и приоритетов как политической, так и экономической направленности [2]. Использование алгоритмов автоматизированной диагностики на основе ИИ может рассматриваться в качестве дополнения к работе врача-диагноста, как «помощника» врача для улучшения качества оказания медицинской помощи.

Основным источником понятий и терминов в сфере планирования и учета инновационной деятельности предприятий, отраслей и стран является 
Руководство Осло [3]. Это нормативный документ Организации экономического сотрудничества и развития (ОЭСР) и Европейского статистического бюро (Евростат), который является основным и признанным в мире. В соответствии с этим документом различают четыре типа инноваций: продуктовые, процессные, маркетинговые и организационные. Интеллектуальная автоматизированная система диагностики (ИАСД) новообразований в легких может считаться универсальной инновацией, так как сочетает в себе признаки всех 4 категорий. Ниже представлено описание системы в соответствии с данными категориями.

Цель работы: разработать план внедрения системы искусственного интеллекта в отделении лучевой диагностики на примере интеллектуальной автоматизированной системы диагностики новообразований в легких «Doctor AIzimov».

Задачи: оценить ИАСД новообразований в легких в соответствии с критериями инновационности. Отразить схему тестового внедрения системы ИИ «Doctor AIzimov» в отделении лучевой диагностики ГБУЗ «СПбКНПЦСВМП(о)». Сформулировать основные шаги в инновационной стратегии внедрения системы ИИ «Doctor AIzimov».

\section{Результаты}

Интеллектуальная автоматизированная система диагностики новообразований

в легких как продуктовая инноващия

Продуктовая инновация есть внедрение товара или услуги, являющихся новыми или значительно улучшенными по части их свойств или способов использования. Сюда включаются значительные усовершенствования в технических характеристиках, компонентах и материалах, во встроенном программном обеспечении, в степени дружественности по отношению к пользователю или в других функциональных характеристиках. Продуктовая инновация в сфере услуг, в частности в медицине, может включать в себя усовершенствования в способах их предоставления (например, эффективности и быстроты) [3].

Как продукт ИАСД Doctor AIzimov - это система поддержки принятия решений врача-рентгенолога, обладает новизной с точки зрения использования за неимением аналогов. На рис. 1 изображена схема системы. Разработанная архитектура реализована на базе облачных сервисов OpenStack, сервера обработки задач Jenkins, библиотек интеграции с планировщиком задач для суперкомпьютера SLURM. Алгоритм использования системы следующий:

1. Получение компьютерных томограмм (КТ) из PACS-системы и сохранение на сервере платформы, установленном в периметре ГБУЗ «СПбКНПЦСВМП(о)» (далее ЛПУ) (на рис. «снимок 1» ... «снимок n» - файлы с данными КТ).

2. Анонимизация снимка КТ - удаление всех персональных данных пациента из метаданных КТ. За периметр ЛПУ отправляются данные КТ с присвоенным уникальным идентификатором без какой-либо информации о пациенте. Анонимизированные результаты обработки сохраняются в базе данных LIRA (http://lira.neurotech.spbstu.ru), доступ к которой на данный момент предоставляется в тестовом режиме по запросу от сторонних организаций при наличии оформленного Соглашения о сотрудничестве.

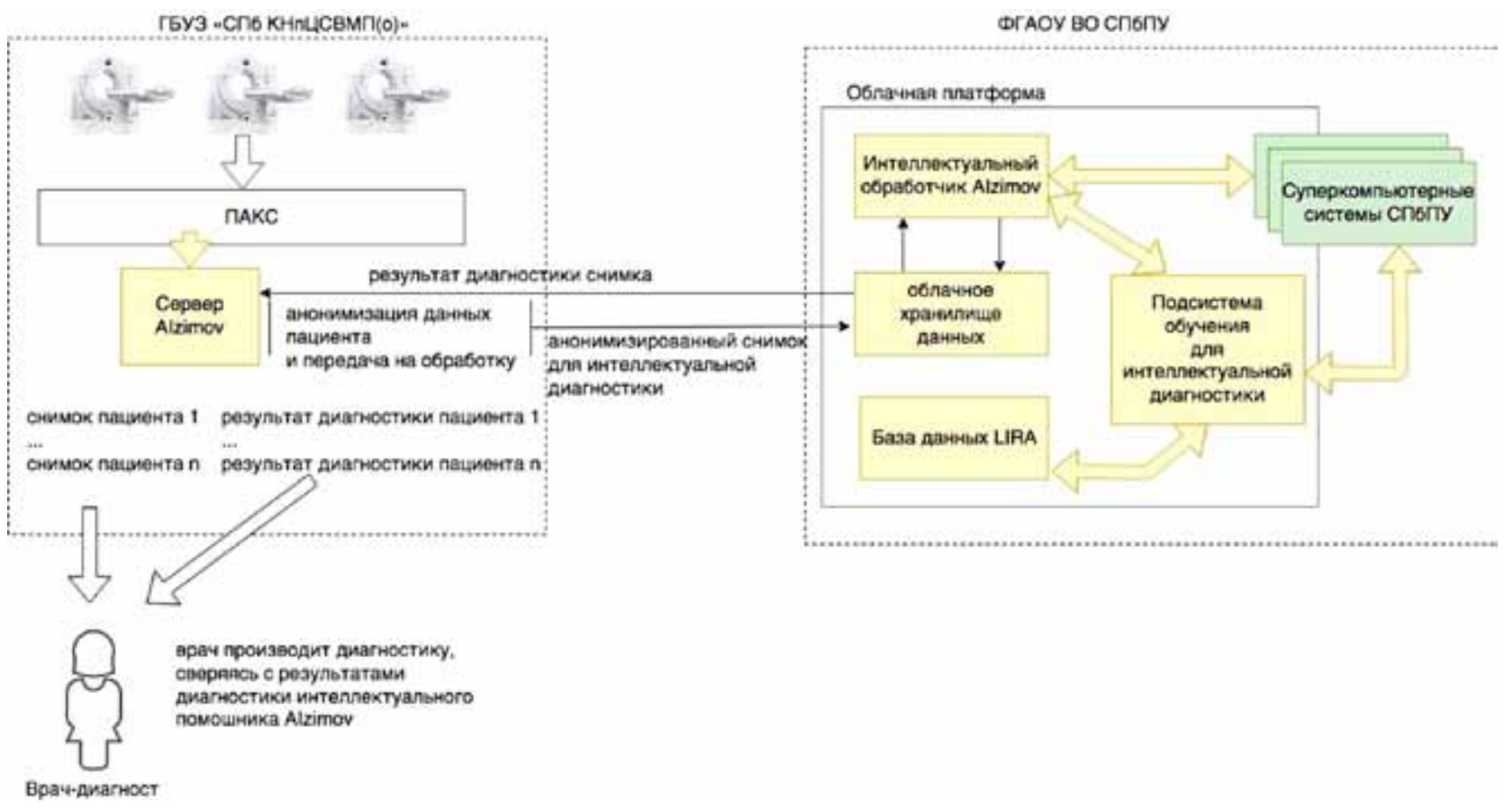

Рис. 1. Схема интеллектуальной автоматизированной системы диагностики новообразований в легких Dосtоr Alzimоv 
3. Передача через облачное хранилище снимка КТ на сервер, установленный в периметре СПбПу.

4. Запуск процесса обработки на вычислительных системах суперкомпьютерного центра СПбПу.

5. Сохранение результатов обработки в облачном хранилище.

6. Получение результатов на сервере, установленном в периметре ЛПу. На рисунке эти данные отмечены как «результат диагностики пациента 1», и другие.

После передачи результатов обработки в ЛПу врач на своем рабочем месте может посмотреть на результаты обработки, сравнить с собственной диагностикой и при необходимости отправить скорректированные результаты в базу данных облачной платформы.

Таким образом, как продуктовая инновация система Doctor AIzimov обладает новизной в свойствах и способе предоставления медицинской услуги, представляя собой предварительный анализ с вынесением «первого мнения», за которым следует анализ снимка врачом. Предварительная обработка диагностической информации ускоряет процесс диагностики врачом.

\section{ИАСД Doctor Alzimov как прочессная инноващия}

Процессная инновация есть внедрение нового или значительно улучшенного способа производства или доставки продукта. Сюда входят значительные изменения в технологии, производственном оборудовании и/или программном обеспечении. Процессные инновации охватывают новые или значительно улучшенные технические приемы, оборудование и программное обеспечение [3].

Процессы, положенные в основу алгоритма Doctor Alzimov, отличаются от имеющихся мировых аналогов тем, что отражают последовательный анализ патологии в легком, приближенный к логике врача. Так обучение системы производится по последовательному представлению признаков опухолевого узла, таким как форма, внутренняя и внешняя структура, причем для понижения размерности обучающих данных используются гистограммы, построенные при обработке изображений. Для представления информации о форме выявляемого объекта применяется метод NEOchord (патент № 2668699, дата приоритета 21 мая 2018 год). Метод основан на построении большого числа отрезков (хорд), соединяющих случайные пары точек на поверхности сегментированного образования. В соответствии с методом, строятся гистограммы длин хорд, которые являются новым представлением признаков объекта по критерию формы с меньшей размерностью [4]. Для уточнения и подтверждения узловой формы объекта дополнительно применялся следующий подход: вокруг объекта достраивался виртуальный куб и строились хорды между внешним контуром объекта и внутренней поверхностью куба. Длины этих новых хорд нормировались по максимальной хорде и так- же строились соответствующие гистограммы. При тестировании системы использование комбинации гистограмм внутренних и внешних хорд повышает точность автоматизированного алгоритма выявления любых новообразований, по форме приближенных к овоидной, на 2,4\% при использовании базы данных LIDC. Для обучения системы по признаку внутренней структуры на каждой внутренней хорде равномерно распределялись $t$ точек, в которых определялись показатели рентгеновской плотности по шкале Хаунсфилда. Здесь $t$ - настраиваемый параметр, зависящий от конкретной реализации системы. В представленном варианте реализации системы плотности определялись в 5 точках каждой хорды. На множестве всех плотностей строилась гистограмма, которая расширяет вектор признаков (гистограмму длин хорд) путем конкатенации.

Именно гистограммы подаются на вход классификатора автоматизированной системы для обучения, таким образом, если данные для обучения системы имеют меньшую размерность, процесс обучения системы упрощается и ускоряется [5]. Так как дифференциальная диагностика РЛ представляет собой отдельную сложную задачу, для алгоритма ИИ Dосtor AIzimov были применены следующие способы реализации:

1. На материале ГБУЗ «СПбКНПЦСВМП(о)» создана уникальная пополняемая база данных LIRA, которая включает подтвержденные морфологически образцы РЛ, патологии, схожей с РЛ, «нетипичных» форм РЛ [6].

2. Использование сиамской нейронной сети (СиамНН). Основная ее идея заключается в том, чтобы обучить две подсети, объединенные на выходе, сравнивать два вектора признаков с точки зрения их семантической близости или различия. Под семантической близостью (различием) понимается принадлежность пары образований одному и тому же диагнозу (различным диагнозам). Таким образом, процесс обучения сети использует все возможные пары образцов, которые имеются в распоряжении, что существенно увеличивает размер обучающей выборки. В процессе эксплуатации пары входных данных для сиамской сети образуются следующим образом. На первый вход подается исследуемый образец неизвестного образования, а на второй - образец из БД LIRA. Если нейронная сеть показала близость этих объектов, то принимается решение о том, что исследуемый образец близок одному из образцов из БД. В противном случае на второй вход подается следующий образец из БД LIRA, и процедура повторяется. Если при сопоставлении исследуемого образца со всеми образцами из БД LIRA во всех случаях сиамская сеть показала отсутствие близости объектов, то принимается решение о том, что исследуемый образец не может быть классифицирован с точки зрения имеющейся информации в БД LIRA и требуется дополнительное обследование. Для понижения размерности данных и ускорения процесса обработки векторы 
признаков также подаются на входы подсетей в виде совокупности гистограмм [7].

Таким образом, осуществляется приближение предлагаемой архитектуры ИАСД Doctor AIzimov к логике врача, который оценивает находку по имеющимся признакам, далее, используя свой опыт, «сравнивает новый объект с виденными ранее».

Исходя из вышеизложенного, следует, что ИАСД Doctor AIzimov имеет признаки процессной инновации, т.к. представляет собой совокупность новых, значительно улучшенных способов реализации систем ИИ, а именно методологии, программного обеспечения.

\section{ИАСД Doctor Alzimov как маркетинговая инноващия}

Маркетинговая инновация есть внедрение нового метода маркетинга, включая значительные изменения в дизайне или упаковке продукта, его размещении, продвижении на рынок или в назначении цены [3]. В медицине маркетинговые инновации могут быть направлены на улучшение удовлетворения нужд потребителя (пациента), то есть качества медицинской помощи, а также на увеличение объема производимых исследований.

Согласно рекомендациям всемирной организации здравоохранения (ВО3), качество медицинской помощи определяется следующими факторами:

- квалификацией персонала и соблюдением им технологий диагностики;
- риском для пациента от медицинского вмешательства;

- наличием и оптимальным использованием ресурсов;

- удовлетворенностью пациента [8].

В России понятие медицинской помощи надлежащего качества раскрыто в нормативных документах. В целом качество медицинской помощи определяется совокупностью характеристик, отражающих своевременность, правильность выбранной тактики и степень достижения результата лечения [9].

Наибольшие трудности определения критериев качества в диагностическом процессе вызывают позиции, связанные с «человеческим фактором», причем показано, что ошибки могут быть связаны с ограничением возможностей метода (30\%), недостатком информации у диагноста (55\%), недостаточной квалификацией врача (15\%) [8]. По данным Национальной академии науки, инженерии и медицины США, диагностические ошибки являются причиной 10\% смертей. Меры по улучшению качества диагностики в концепции этой организации направлены в первую очередь на следующие моменты:

- улучшение эффективности командной работы среди медицинских работников и самих пациентов;

- укрепление профессионального образования;

- информационные медицинские технологии [10].

Разработанная система диагностики образований в лёгких может отвечать всем вышеуказанным пози-

Оценка эффективности использования системы ИИ

Таблища 1.

\section{в соответствии с критерияии качества медицинской помощи}

\begin{tabular}{|c|c|c|}
\hline Критерий качества & Система Ии & Специалист \\
\hline Своевременность & $\begin{array}{l}\text { Время обработки данных КТ на про- } \\
\text { цессоре і5 с частотой } 1,7 \text { Гц занимает } \\
\text { максимум } 42 \text { секунды. }\end{array}$ & $\begin{array}{l}\text { Среднее время описания КТ грудной клет- } \\
\text { ки онкологического пациента } 20 \text { минут. } \\
\text { Регламентированное время выдачи за- } \\
\text { ключения } 24 \text { часа [11]. }\end{array}$ \\
\hline «Командная работа» & $\begin{array}{l}\text { Использование системы возможно как } \\
\text { в качестве первичного анализа, с по- } \\
\text { следующей интерпретацией врачом, } \\
\text { так и в качестве проверки заключения } \\
\text { врача. }\end{array}$ & Зачастую отсутствует второе мнение. \\
\hline Укрепление образования & $\begin{array}{l}\text { Обучение системы на основе большого } \\
\text { количества входных данных. } \\
\text { В системе Doctor AIzimov использу- } \\
\text { ются алгоритмы на основе глубоких } \\
\text { лесов, что обладает воспроизводи- } \\
\text { мостью результатов [4], поэтому врач } \\
\text { может укреплять свои знания, изучив } \\
\text { алгоритм принятия решения системой. }\end{array}$ & $\begin{array}{l}\text { Ограниченное количество специалистов } \\
\text { высшей категории, недостаток кадров в } \\
\text { регионах, несовершенная система пост- } \\
\text { дипломного образования, отсутствие си- } \\
\text { стемы анализа диагностических ошибок. }\end{array}$ \\
\hline $\begin{array}{l}\text { Наличие } \\
\text { и оптимизация ресурсов }\end{array}$ & $\begin{array}{l}\text { Нет зависимости от имеющегося в ЛПУ } \\
\text { томографа. } \\
\text { Корректировка пропускной способ- } \\
\text { ности кабинета КТ. }\end{array}$ & $\begin{array}{l}\text { Пропускная способность зависит от } \\
\text { штатного расписания, при этом время на } \\
\text { интерпретацию превышает время самого } \\
\text { исследования в 3-4 раза. }\end{array}$ \\
\hline
\end{tabular}


циям на пути к улучшению качества диагностической медицинской помощи (табл.1).

Из сравнительной таблицы видно, что ИАСД Dосtor Alzimov имеет признаки маркетинговой инновации, поскольку измененный дизайн проводимого обследования направлен на удовлетворение нужд пациента с позиции показателей качества медицинской помощи.

Организационная инновация есть внедрение нового организационного метода в деловой практике предприятия, в организации рабочих мест или внешних связей. Отличием организационной инновации от простых организационных изменений является внедрение какого-либо ранее не использовавшегося организационного метода, который является результатом реализации стратегических решений руководства [3]. Организационные инновации в диагностике должны подразумевать внедрение не только новых методов в организацию повседневной деятельности врача-рентгенолога, но и может повлечь изменениереорганизацию работы отделения лучевой диагностики. В работе [12] отражены основные направления, которые могут быть основой для внедрения ИИ в деятельность медицинского учреждения с организационной точки зрения:

1. Для реализации любой инновационной стратегии необходима перестройка организации или ее реструктуризация в сочетании с текущими производственными процессами с учетом включения в неё инновационной составляющей.

2. Инновационная стратегия должна содержать ясные директивы и временные ориентиры, быть гибкой, поддерживаться организационным дизайном и коррелировать с общей корпоративной стратегией.

3. Инструментом преодоления неопределённости в стратегии является экспериментирование.

4. Процесс установления целей инновационной стратегии преобразует стратегическое видение в те результаты, к которым надо стремиться.

5. Результат инновационных изменений должен быть ориентирован на потребности потребителя (пациента).

6. Организационные и технологические инновации являются одновременно причиной и следствием постоянного усовершенствования компании.

Внедрение алгоритма ИИ как организационной инновации представляется наиболее сложной задачей в связи с выраженным консерватизмом врачебного сообщества, ограниченным информационным полем для развития инновационной культуры, нежеланием перемен. Основным препятствием можно считать ошибочное мнение о том, что специальность рентгенология «изжила себя», специалисты будут не нужны и начнется резкое сокращение кадров. По мнению авторов, напротив, включение алгоритма ИИ в процесс анализа снимка КТ может стать новой практикой для улучшения обучения сотрудников и обмена знаниями внутри медицинского сообщества. Применение алгоритма на основе глубокого леса и сиамской нейронной сети в системе Doctor Alzimov обусловливает интерпретируемость и воспроизводимость результата. В ряде работ, посвященных развитию ИИ в медицине, показаны основные недостатки алгоритмов на основе нейронных сетей (НC), которые связаны с вычислительной сложностью и закрытостью (то есть на выходе НСмы не можем знать, как делается тот или иной вывод о заболевании, как выглядит обученная функция НС, как осуществляется обработка информации) [13]. Таким образом, для применения в медицине, где выборки обучающих и тестирующих данных априори невелики, использование алгоритма на основе глубоких лесов представляется более оправданным $[14,15]$. СиамНН последовательно сравнивает новое образование со всеми образованиями из БД. Аналогичным образом действует и врач, когда анализирует изображение КТ, используя свой опыт ранее анализируемых изображений. Кроме того, получив определенное решение с использованием ИАСД, врач может всегда его проверить путем анализа изображения КТ из БД, которое оказалось наиболее близким к новому образованию. Именно это свойство Doctor AIzimov обусловливает интерпретируемость и именно оно отсутствует в большинстве систем, использующих обычные нейронные сети для классификации изображений КТ.

Ограничением данного подхода может быть только недостаточное количество образцов в базе данных, однако данный недостаток может быть преодолен в процессе постоянного пополнения базы данных в течение рутинной работы отделения лучевой диагностики.

Реализация организационной инновации Dосtor AIzimov в отделении лучевой диагностики ГБУз «СПбКНПЦСВМП(о)» была осуществлена в процессе закрытого тестирования системы, которое способствовало появлению интересной схемы взаимодействия ИИ - врач. В этом качестве у рентгенолога появляется новый уровень приложения знаний. А именно внедрение практики кодификации знаний, т.е. практика постоянного обновления базы данных при выявлении каждого нового случая заболевания. На первоначальном этапе использования системы Doctor AIzimov врач на своем рабочем месте получает обработанные системой данные и сравнивает результат со своей собственной интерпретацией патологии. Заключение выносится врачом. После морфологической верификации процесса врач кодирует результат, направляет снимки на дообучение системы или на пополнение базы данных в зависимости от того, верно ли система распознала результат до верификации. Выявленные новые случаи отправляются на дообучение системы, что будет приводить к ее постоянному совершенствованию. Данный подход также может быть использован также и в подборе учебного материала 
таким образом, чтобы сделать его более доступным для персонала. Таким образом, будет происходить одновременное «взаимовыгодное» дообучение интеллектуальной системы и повышение квалификации врача-рентгенолога. Кроме того, таким образом будет совершенствоваться управление качеством ИИ, так как в процессе вовлечена работа мультидисциплинарной команды специалистов и ИИ. Врач-рентгенолог приобретает новый статус "специалиста по данным», управляя диагностическими процессами. Вероятен вариант развития событий, что с ростом количества цифровой информации и совершенствованием систем ИИ функциональные обязанности рентгенолога и его профессиональные навыки будут сдвигаться в сторону «кодификации знаний», то есть врач будет реализовывать свой потенциал на новом уровне, определяя новые задачи для ИИ.

Исходя из изложенного, место системы ИИ в диагностике рака легкого Doctor Alzimov соответствует критериям инновационности. Конкурентными преимуществами системы является ее реализация без зависимости от модели компьютерного томографа, система может быть использована на любом компьютере или планшете. Таким образом, данное положение обусловливает актуальность разработки инновационной стратегии внедрения систем ИИ в медицине при развитии инновационной культуры в медицинском сообществе и отсутствии стагнации.

\section{План внедрения системы ИИ Doctor Alzimov в работу отделения лучевой диагностики специализированного учреждения}

Особенностью внедрения системы ИИ является то, что она обладает признаками всех категорий инновационности. Это должно быть учтено при планировании соответствующих мероприятий.

Внедрение системы как продукта должно быть подтверждением ориентированности ее в интересах пациента, что подразумевает разработку основ «объяснимости», под которыми подразумевается четкое изложение в доступных источниках основ, целей использования ИИ, преимуществ для пациента, таких как повышение качества диагностики и безопасность. Меры по информированию пациентов о новой структуре диагностики призваны устранить барьер недоверия у пациента.

Внедрение системы как процесса подразумевает определение и прописывание нового диагностического алгоритма работы врача-рентгенолога, у которого появляются новые функциональные задачи, которые связаны с кодификацией знаний, получением «обратной связи» по результатам морфологической верификации образования в легком.

Внедрение системы ИИ с точки зрения маркетинговой инновации означает, что необходимо проведение мер по перерасчету норм нагрузки на врача и пропускной способности компьютерного томографа, пересмотр ключевых показателей эффективности отделения лучевой диагностики.

Для внедрения организационной инновации системы Doctor AIzimov предлагается выделить экспериментальные кадровые позиции для врачей рентгенологов, чтобы оценить организационную инновацию по критериям ключевых показателей эффективности. В то же время междисциплинарное взаимодействие врач - data scientist будет способствовать усовершенствованию системы ИИ и ее дообучению в процессе текущей работы. При этом, мера ответственности за диагностику сохраняется за врачом, что не противоречит нормативно-правовой базе, устанавливается мера ответственности за обучение системы ИИ для data scientist. При этом возможно смещение функциональных навыков врача-рентгенолога в сторону узкой специализации в зависимости от направленности разрабатываемых систем ИИ.

\section{Выводы}

Системы ИИ в медицине, в частности ИАСД Dосtor AIzimov, обладают всеми критериями инноваций со значительным уровнем новизны как рынка так и научного потенциала, носят комплексный характер с точки зрения классификации инноваций.

C внедрением ИАСД Doctor AIzimov врачрентгенолог приобретает новые навыки по «кодификации» знаний, при этом мера его ответственности за диагностику не меняется.

Мультидисциплинарный обмен информацией между врачом и разработчиком систем ИИ (data scientist) является инновационным сотрудничеством, что дает возможность получать и использовать знания, которыми каждый из участников не мог бы воспользоваться самостоятельно.

Внедрение инновации - системы ИИ Doctor AIzimov в деятельность специализированного медицинского учреждения направлено на удовлетворение потребностей пациента, коррелирует с общей государственной и корпоративной стратегией, не противоречит общему устоявшемуся дизайну диагностического процесса, имеет под собой конкретные цели, является одновременно причиной и следствием постоянного усовершенствования деятельности учреждения в целом, и отделения лучевой диагностики в частности.

\section{Список литературы}

1. Сыстерова А.А., Тоцкая Е.Г. Внедрение инноваций в здравоохранении. // Проблемы социальной гигиены, здравоохранения и истории медицины: научно-практический журнал. - 2012. - № 5. - С. 17-18.

2. Указ Президента Российской Федерации от 9 мая 2017 г. № 203 о Стратегии развития информационного общества в Российской Федерации на 2017-2030 годы. 
3. Oslo Manual: Guidelines for Collecting and Interpreting Innovation Data, 3rd Edition@ OECD/EC, 2005. // Перевод на русский язык. Государственное учреждение «Центр исследований и статистики науки» (ЦИСН). - 2006. - 192 с.

4. Моисеенко В.М., Мелдо А.А., Уткин Л.В., Прохоров И.Ю., Рябинин М.А., Богданов А.А. Автоматизированная система обнаружения объемных образований в легких как этап развития искусственного интеллекта в диагностике рака легкого. // Лучевая диагностика и терапия. - 2018. - № 3. - С. 62-68.

5. Мелдо А.А., Уткин Л.В., Трофимова Т.Н., Рябинин М.А., Моисеенко В.М., Шелехова К.В. Новые подходы к разработке искусственного интеллекта в диагностике рака легкого. // Лучевая диагностика и терапия. - Принята к печати.

6. Прохоров И.Ю., Рябинин М.А., Мелдо А.А., Уткин Л.В. Формирование баз данных с целью машинного обучения в диагностике рака легкого. // Конгресс Российского общества рентгенологов и радиологов. Сборник тезисов. - 2018. - СПб. - С. 124-125.

7. Meldo A.A., Utkin L.V. A computer-aided system for differential diagnosis of lung diseases. // Intelligent Data Processing: Theory and Applications. Book of abstracts of the $12^{\text {th }}$ International Conference (Moscow, Russia - Gaeta, Italy, 2018). - Moscow: TORUS PRESS. - 2018 - P. 35.

8.Мелдо А.A. Методика разработки критериев качества в условиях отделения лучевой диагностики бюджетного медицинского учреждения. // Лучевая диагностика и терапия. - 2017. - Т. 3, № 8. - С. 5-10.

9. Приказ Минздрава России от 7.07.2015 № 422ан «Об утверждении критериев оценки качества медицинской помощи".

10. Balogh E.P., Miller B.T., Ball J.R. Improving diagnosis in health care. // The National Academies press. Washington. - 2015. - $444 \mathrm{p}$.

11. Приказ Минздрава РФ от 2 августа 1991 г. № 132 «О совершенствовании службы лучевой диагностики».

12. Шилович А. Инновационная деятельность и её основные направления. // Планово-экономический отдел. - 2004. - № 4. - С. 10.

13. Bramer M. Principles of Data Mining. - Springer. - 2007. - 354 p.

14. Zhou Z.-H., FengJ. Deep forest: Towards an alternative to deep neural networks. // Proceedings of the 26th International Joint Conference on Artificial Intelligence (IJCAI'17). - Melbourne: Australia. - 2017. - P. 3553-3559.

15. Utkin L.V., Ryabinin M.A. A Siamese deep forest. // Knowledge-Based Systems. - 2018. - Vol. 139. - P. 13-22

\section{References}

1. Systerova A.A., TotskayaYe.G. The implementation of innovations in public health. Problems of social hygiene, health care and the history of medicine: scientific and practical journal. 2012; 5: 17-18. (In Russ)

2. Presidential Decree of May 9, 2017 No. 203 on the Strategy for the Development of the Information Society in the Russian Federation for 2017-2030. (In Russ)

3. Oslo Manual: Guidelines for Collecting and Interpreting Innovation Data, 3rd Edition @ OECD/EC. 2005: 192.

4. Moiseyenko V.M., Meldo A.A., Utkin L.V., Prokhorov I.Yu., Ryabinin M.A., Bogdanov A.A. Automated detection system for lung masses as a stage of artifitial intelligence development in the diagnostics of lung cancer. Diagnostic radiology and radiotherapy. 2018; (3): 62-68. (In Russ.) doi: 10.22328/2079-5343-2018-9-3-62-68.

5. Meldo A.A., Utkin L.V., Trofimova T.N., Ryabinin M.A., Moiseenko V.M., Shelekbova K.V. Novel approaches to development of artificial intelligence algorithms in the lung cancer diagnostics. Diagnostic radiology and radiotherapy. 2019; (1): accepted for printing. (In Russ)

6.Prokborov I.Yu., Ryabinin M.A., Meldo A.A., Utkin L.V. Formation of databases for the purpose of machine learning in the diagnosis of lung cancer. Congress of the Russian Society of Radiologists and Radiologists. Collection of theses. 2018; 124-125. (In Russ)

7. Meldo A.A., Utkin L.V. A computer-aided system for differential diagnosis of lung diseases. Intelligent Data Processing: Theory and Applications. Book of abstracts of the $12^{\text {th }}$ International Conference (Moscow, Russia - Gaeta, Italy, 2018). - Moscow: TORUS PRESS. 2018; 35.

8. Meldo A.A. Development and evaluation of quality management processes in the radiology department of government specialized clinic. Diagnostic radiology and radiotherapy. 2018; (1): 5-10. (In Russ.) doi: 10.22328/20795343-2018-9-1-5-10.

9. Order of the Ministry of Health of Russia dated 07.07.2015 No. 422an «On approval of criteria for assessing the quality of medical care». (In Russ)

10. Balogh E.P., Miller B.T., Ball J.R. Improving diagnosis in health care. The National Academies press. Washington. $2015 ; 444$.

11. Order of the Ministry of Health of the Russian Federation of August 2, 1991 No. 132 «On the improvement of the radiological diagnostics service». (In Russ)

12. Shilovich A. Innovative activity and its main directions. Planning and Economic Department. 2004; (4): 10. (In Russ)

13. Bramer M. Principles of Data Mining. Springer. 2007; 354.

14.Zhou Z.-H., FengJ. Deep forest: Towards an alternative to deep neural networks. Proceedings of the 26th International Joint Conference on Artificial Intelligence (IJCAI'17). Melbourne: Australia. 2017; 3553-3559.

15. Utkin L.V., Ryabinin M.A. A Siamese deep forest. Knowledge-Based Systems. 2018; (139): 13-22. 